\title{
TURBULENT SWIRLING FLOW IN A CIRCULAR PIPE
}

\author{
Shiro ITO, Kohei OGAWA and ChIAKi KURODA \\ Department of Chemical Engineering, \\ Tokyo Institute of Technology, Tokyo 152
}

\begin{abstract}
The decay process of turbulent swirling flow in an axisymmetric circular pipe is analyzed and a method to estimate the tangential mean velocity distribution is proposed.

Two basic new factors, viz. the imaginary inlet position of the swirl decay and the friction velocity $U_{\theta f}$ for the swirling flow, were defined in order to investigate the distribution of the intensity of swirl $\boldsymbol{\Gamma}$. It became clear that there were characteristic functional relationships between the dimensionless radius $r^{+}$and the dimensionless intensity of swirl $\Gamma^{+}$in the forced vortex zone and in the quasi-free vortex zone, respectively, where $r$ and $\Gamma$ were made dimensionless by $U_{\theta f}$. By making use of these relationships between $r^{+}$and $\Gamma^{+}$and the exponential decay process of $\Gamma$ in the axial direction after determination of the values of empirical coefficients, a calculation procedure for tangential mean velocity in a given condition is proposed.
\end{abstract}

\section{Introduction}

Swirling flow can be seen in many kinds of chemical equipment, such as cyclone separators and stirred vessels, and it can be considered that swirling flow dominates the performance of such equipment. When its performance is studied, it becomes an important matter how to estimate the velocity distributions. In general, swirling flows are turbulent, and no clear method of estimating even the tangential velocity distributions has been established until now. A few papers ${ }^{5-9}$ have considered this problem based on the results of numerical analyses involving many unreal assumptions or on only experimental results.

The purpose of this paper is to make clear the distribution form of the tangential mean velocity in steady axisymmetric turbulent swirling flow by using the three following kinds of information; the distributions of general statistical quantities of the velocity fluctuation, the decay of the swirl intensity and a modified concept of the law of the wall which is often used in the investigation of the turbulent boundary layer.

\section{Estimation of Tangential Velocity Distribution}

It is possible to express the tangential velocity by the intensity of swirl based on the concept of circulation $^{4}$. That is, in steady axisymmetric swirling flow of an incompressible fluid, the following relationship between the intensity of swirl $T$ and the tangential velocity $U_{\theta}$ holds:

$$
\Gamma \equiv \iint_{S}(\boldsymbol{\Omega} \cdot \boldsymbol{n}) d S=\oint_{C}(\boldsymbol{U} \cdot \boldsymbol{t}) d C=2 \pi r U_{\theta}
$$

\footnotetext{
Received April 4, 1979. Correspondence concerning this article should be addressed to C. Kuroda. S. Ito is now with JGC CORPORATION, Tokyo 100
}

where $S$ is the circular cross-sectional area perpendicular to the pipe axis, $\Omega$ the vorticity vector, $\boldsymbol{n}$ the unit normal vector to $S, C$ the circular closed curve surrounding $S, U$ the velocity vector, $\boldsymbol{t}$ the unit tangential vector along $C$. Then the tangential velocity can be treated through the intensity of swirl.

\section{1 Axial decay of $\Gamma$}

It has already been confirmed ${ }^{4}$ that the axial decay of $\Gamma$ can be expressed by the exponential function of the dimensionless factor $(Z / R)\left(\nu / R^{2} \omega_{i}\right)$, where $Z$ is the distance between the measured section and the inlet section, $R$ the inner pipe radius and $\omega_{i}$ the initial angular velocity at the inlet position $(Z=0 \mathrm{~cm})$. The cross-sectional overall intensity of swirl $\Gamma_{w}(=2 \pi$ $\left.R U_{\theta w}\right)$ is assumed to be expressed as

$$
\Gamma_{w}^{*}=\frac{\Gamma_{w}}{2 \pi R^{2} \omega_{i}}=\gamma \exp \left\{-\kappa\left(\frac{Z}{R}\right)\left(\frac{\nu}{R^{2} \omega_{i}}\right)\right\}
$$

where $\Gamma_{w}^{*}$ is the dimensionless quantity of $\Gamma_{w}$ and $U_{\theta w}$ is the extrapolated value of $U_{\theta}$ at the pipe wall in the quasi-free vortex zone of the swirling flow.

Here, as shown by Eq. (1), $\Gamma$ is equivalent to the integrated value of the axial vorticity component $\omega_{x}$ over the cross-sectional circular area. Since the value of $\omega_{z}$ is much smaller in the quasi-free vortex zone than that in the forced vortex zone $\left(\omega_{z}\right.$ is zero in the ideal free vortex), the value of $\Gamma_{w}$ may be considered to depend on the value inside the forced vortex zone. Consequently, when the intensity of swirl in the forced vortex zone is shown as $\Gamma_{b}$, it is expected that there will be a characteristic functional relationship between $\Gamma_{b}^{*}\left(=\Gamma_{b} / 2 \pi R^{2} \omega_{i}\right)$ and $(Z / R)\left(\nu / R^{2} \omega_{i}\right)$ in the same manner as in the case of $\Gamma_{w}^{*}$.

\subsection{Radial distribution of $\Gamma$}

It was confirmed ${ }^{3)}$ experimentally that the distribu- 
tion form of turbulent statistical quantities in the forced vortex zone is distinguished from that in the socalled quasi-free vortex zone. In particular, the Reynolds stress $-\rho \overline{u_{r} u_{\theta}}$ takes zero value in the vicinity of the boundary position between the above-mentioned two zones. Since the boundary position between the two zones is expected to be the characteristic position from the viewpoint of the turbulence mechanism, let the radial position at which $U_{\theta}$ takes the maximum value $U_{\theta b}$ be $r_{b}$ as the representative boundary position between two zones, as shown in Fig. 1. Then the Reynolds stress $-\rho \overline{u_{r} u_{\theta}}$ takes zero value at $r_{b}$.

A hypothetical cylindrical wall is assumed to be situated at $r_{b}$ so that the concept of the law of the wall can be used. That is, the shear stress $\tau_{r \theta, b}$ at $r_{b}$ is equivalent to the shear stress at the hypothetical wall and the following representative velocity is defined.

$$
U_{\theta f} \equiv \sqrt{\frac{\left|\tau_{r \theta, b}\right|}{\rho}}
$$

The shear stress of Newtonian fluids in axisymmetric turbulent flow is written as

$$
\tau_{r \theta}=\mu\left(\frac{\partial \bar{U}_{\theta}}{\partial r}-\frac{\bar{U}_{\theta}}{r}\right)-\rho \overline{u_{r} u_{\theta}} .
$$

The shear stress at $r_{b}$ is shown as the following simple form:

$$
\tau_{r \theta, b}=-\mu\left(\frac{U_{\theta b}}{r_{b}}\right)
$$

because the values of both $\partial \bar{U}_{\theta} / \partial r$ and $\overline{u_{r} u_{\theta}}$ can be considered to be zero from the experimental results. Some examples of relationships between $r / r_{b}$ and $\widehat{u_{r} u_{\theta}} / U_{\theta b}^{2}$ are shown in Fig. 2. Consequently, Eq. (3) can be rewritten as

$$
U_{\theta f}=\sqrt{\frac{\nu U_{\theta b}}{r_{b}}}
$$

In this study, $U_{\theta f}$ is applied to the transformation of the radius $r$ and the intensity of swirl $\Gamma$ into dimensionless quantities $r^{+}$and $\Gamma^{+}$respectively in order to investigate the radial distribution of $\Gamma$.

$$
\begin{gathered}
r^{+} \equiv \frac{U_{\theta f}}{\nu} r=\sqrt{\frac{\bar{U}_{\theta b}^{-}}{\nu r_{b}} r} \\
\Gamma^{+} \equiv \frac{\Gamma}{2 \pi r_{b} U_{\theta f}}=\frac{r \bar{U}_{0}}{\sqrt{\nu r_{b} U_{\theta b}}}
\end{gathered}
$$

The relation between $r^{+}$and $\Gamma^{+}$should be investigated experimentally.

Here, if the radial distribution of $\Gamma$ can be altogether represented, $U_{\theta}$ at a given position can be estimated by introducing the information about the axial decay of $\Gamma$ in the section 1.1.

\section{Experimental Apparatus and Procedure}

The experimental apparatus is shown in Fig. 3.

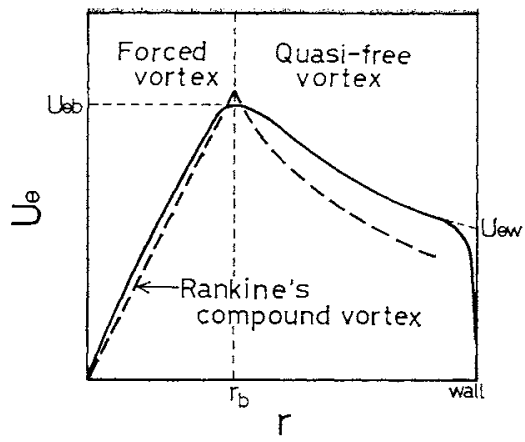

Fig. 1 Tangential velocity distribution

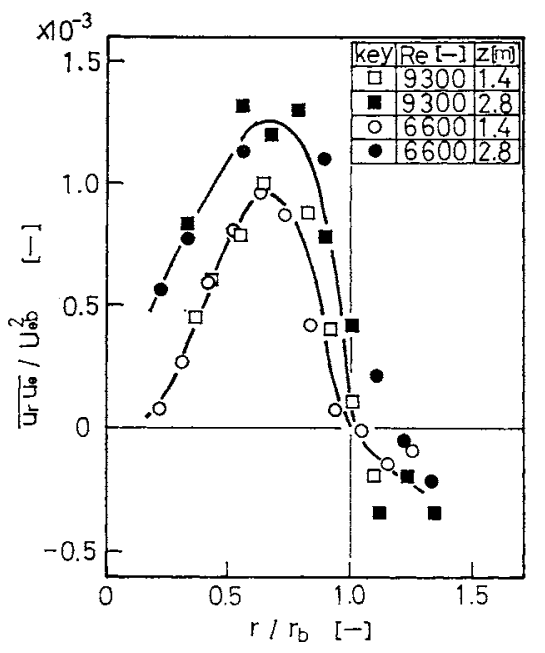

Fig. 2 Reynolds stress $-\rho \overline{u_{r} u_{\theta}}$ distribution

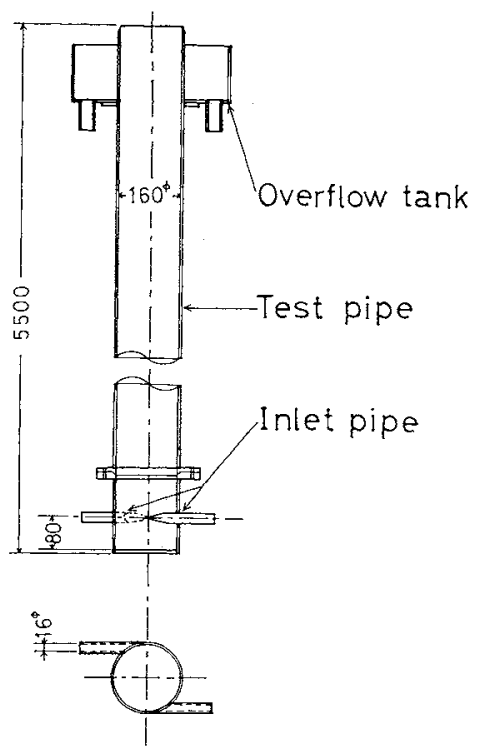

Fig. 3 Experimental apparatus

The test circular pipe used in this experiment was a smooth, vertical acrylic pipe of $160 \mathrm{~mm}$ inner diameter and $5.5 \mathrm{~m}$ length. Two inlet pipes of $16 \mathrm{~mm}$ inner diameter were attached tangentially at $80 \mathrm{~mm}$ height from the bottom of the test pipe, and the test fluid was injected into the test pipe through the inlet 


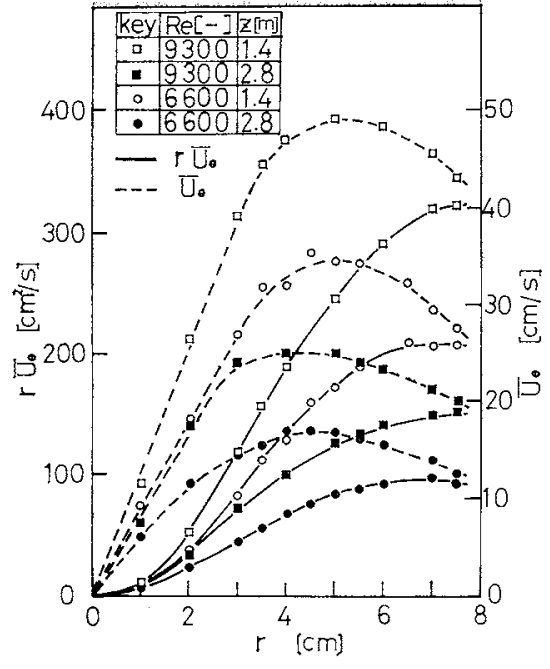

Fig. 4 Tangential velocity and circulation distributions

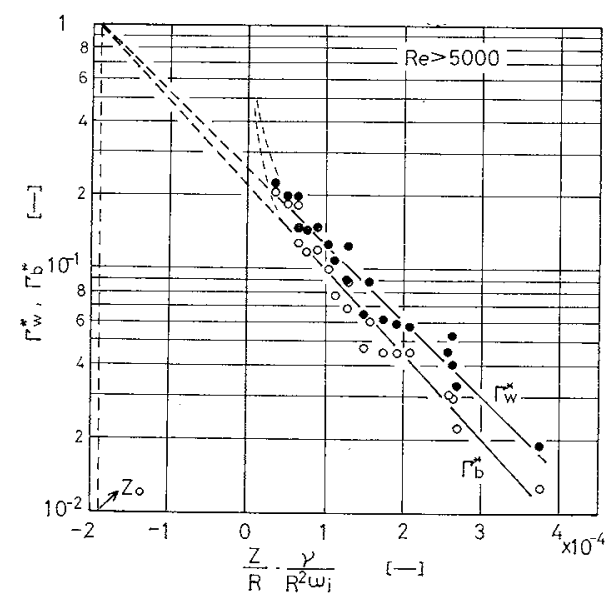

Fig. 5 Decay of intensity of swirl

pipes at an equal flow rate, creating an almost completely axisymmetric flow.

The test fluid was an electrolytic solution containing $\mathrm{K}_{3} \mathrm{Fe}(\mathrm{CN})_{6}, \mathrm{~K}_{4} \mathrm{Fe}(\mathrm{CN})_{6}\left(3.0 \times 10^{-3} \mathrm{~mol} / \mathrm{l}\right.$, respectively) and the supporting electrolyte $\mathrm{KCl}\left(1.0 \times 10^{-1}\right.$ $\mathrm{mol} / l$ ) to measure the flow velocity electrochemically. The value of its kinematic viscosity is nearly equal to that of water.

To measure the three-dimensional velocity components, a spherical multi-electrode probe ${ }^{1,2)}$ of diameter $7.9 \mathrm{~mm}$ was used.

The measurement was carried out at $70,140,210,280$, 350 and $500 \mathrm{~cm}$ height from the inlet section. The flow was set at the completely turbulent condition as $\operatorname{Re}\left(=D U_{\mathrm{a} v} / \nu\right)>5000$.

\section{Results and Discussion}

Some examples of the radial distributions of $\bar{U}_{0}$ and $r \bar{U}_{\theta}$ are shown in Fig. 4. From these data, the distribution of the intensity of swirl $\Gamma$ was investigated as follows.

\section{1 Axial decay of $\dot{T}$}

The correlations between $\Gamma_{w}^{*}$ or $\Gamma_{b}^{*}$ and $(Z / R)$ $\times\left(\nu / R^{2} \omega_{i}\right)$ are shown in Fig. 5. Each correlation is expressed as follows.

$$
\Gamma_{w}^{*}=0.26 \exp \left\{-7100(Z / R)\left(\nu / R^{2} \omega_{i}\right)\right\}
$$

and

$$
\Gamma_{b}^{*}=0.22 \exp \left\{-8000(Z / R)\left(\nu / R^{2} \omega_{i}\right)\right\}
$$

From the experimental condition, $\Gamma_{w}^{*}$ is expected to approach unity as $Z$ approaches zero. The reason is that the initial angular velocity $\omega_{i}$ is defined as $U_{i} / R$ and that $U_{\theta w}$ is expected to become nearly $U_{i}$ at the inlet position. However, as is evident from Fig. 5, Eq. (9) cannot express the decay of $I_{w}^{*}$ near the inlet region. But, assuming that the real decay near the inlet region, which is considered to be influenced by the inlet structure, is ignored, the imaginary inlet position $\left(-Z_{0} / R\right)\left(\nu / R^{2} \omega_{i}\right)$ can be defined at the position where $\Gamma_{w}^{*}$ is unity by extrapolating the line of Eq. (9) in the negative direction of $(Z / R)\left(\nu / R^{2} \omega_{i}\right)$.

As shown in Fig. 5, if the straight line of Eq. (10) is extrapolated in the negative direction of $(Z / R)(\nu /$ $\left.R^{2} \omega_{i}\right)$ in the same manner, the position where $\Gamma_{b}^{*}$ is unity agrees with that for $\Gamma_{w}^{*}$. Therefore, the assumption that the forced vortex $\left(U_{\theta}=\omega_{i} r\right)$ extends to the pipe wall in the cross-section of the imaginary inlet position will be reasonable.

The above-defined dimensionless imaginary inlet position $\left(-Z_{0} / R\right)\left(\nu / R^{2} \omega_{i}\right)$ is expected to be one basic position of this experimental apparatus.

\section{2 Radial distribution of $\Gamma$}

The relation between $r^{+}$and $\Gamma^{+}$is to be investigated in the forced vortex zone and the quasi-free vortex zone, respectively. That is, by making use of $r_{b}^{+}$and $\Gamma_{b}^{+}$at the boundary position, the relation between $r_{b}^{+}-r^{+}$and $\Gamma_{b}^{+}-\Gamma^{+}$is investigated in the forced vortex zone of $r<r_{b}$, and the relation between $r^{+}-$ $r_{b}^{+}$and $\Gamma^{+}-\Gamma_{b}^{+}$is investigated in the quasi-free vortex zone of $r>r_{b}$. These relationships are shown in Fig. 6. As is evident from this figure, a characteristic functional relationship is obtained in each zone, and the form of the function is approximately as follows.

$$
\begin{aligned}
& \Gamma_{b}^{+}-\Gamma^{+}=\left(r_{b}^{+}-r^{+}\right)^{1.06} \\
& \quad \text { for the forced vortex zone } \\
& \Gamma^{+}-\Gamma_{b}^{+}=\left(r^{+}-r_{b}^{+}\right)^{0.92} \\
& \quad \text { for the quasi-free vortex zone }
\end{aligned}
$$

In $r^{+}-r_{b}^{+} \simeq 40-70$ of Fig. 6 (b), some measured points in the vicinity of the pipe wall deviate slightly from Eq. (12).

\section{3 Estimation of tangential velocity $U_{\theta}$}

When the value of the tangential velocity $U_{0}$ at a position $(r, z)$ must be known under given conditions, one can obtain the value by the following way 

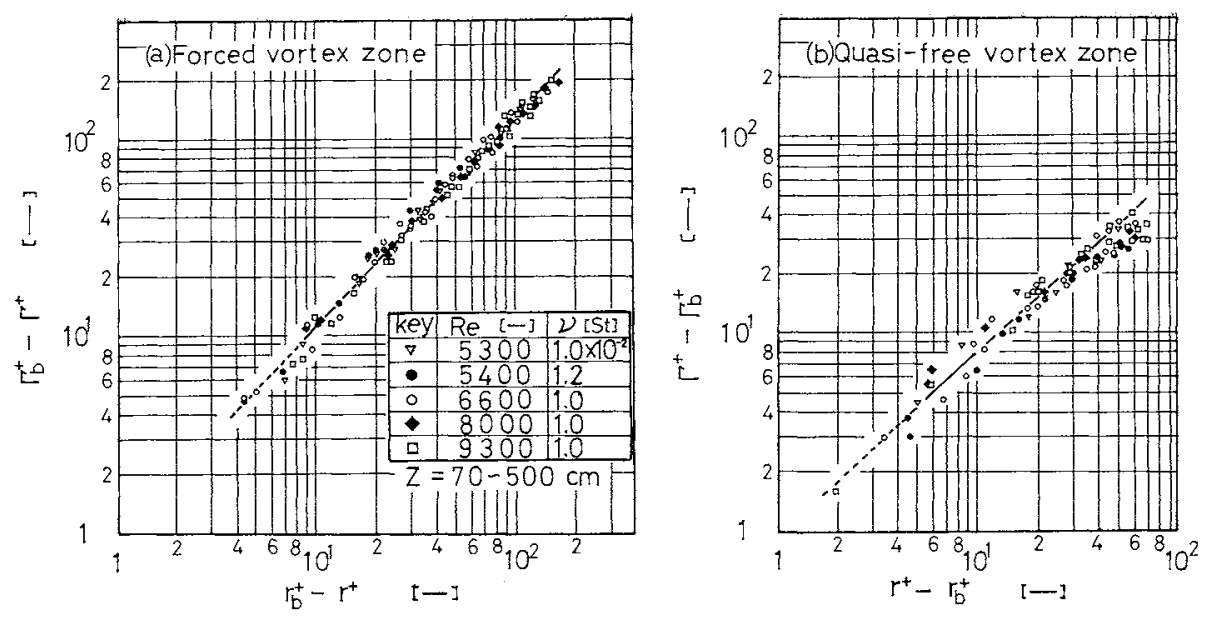

Fig. 6 Relationship between $r^{+}$and $\Gamma^{+}$

(see Fig. 7). First, the values of $\Gamma_{w}^{*}$ and $\Gamma_{b}^{*}$ are calculated from the value of $(Z / R)\left(\nu / R^{2} \omega_{i}\right)$ by Eqs. (9) and (10), and consequently $R U_{\theta w}$ and $r_{b} U_{\theta b}$ can be obtained. Secondly, the value of $\Gamma_{w}^{+}-\Gamma_{b}^{+}$is calculated from the values of $R U_{\theta w}$ and $r_{b} U_{\theta b}$ by Eq. (8). Thirdly, the value of $R^{+}-r_{b}^{+}$is calculated by Eq. (12). Since $r_{b}^{+}$is expressed as $r_{b} U_{\theta f} / \nu=\left(r_{b} U_{\theta b} / \nu\right)^{1 / 2}$ and the value of $r_{b} U_{0 b}$ has already been obtained, the value of $R^{+}$is calculated and consequently $U_{\theta f}$ can be obtained by Eq. (7). Finally, the value of $U_{\theta}$ at $(r, z)$ can be obtained from the value of $\Gamma^{+}$which is calculated by Eq. (11) or (12) and Eq. (7). This calculation procedure is applicable except in the close vicinity of the inlet region, the pipe wall and the center axis.

When studying swirling flows in other equipment, the values of empirical coefficients in equations such as $\gamma$ in Eq. (2) have to be determined anew. However, the above-mentioned procedure of calculation is expected to be applicable in the same manner to other turbulent swirling flows in a circular pipe.

\section{Conclusions}

The conclusions are summarized as follows:

1) Two important new basic factors for turbulent swirling flow are defined. One is the imaginary inlet position of the swirl decay and the other is the friction velocity for the swirling flow.

$$
U_{\theta f} \equiv\left(\left|\tau_{r \theta, b}\right| / \rho\right)^{1 / 2}=\left(\nu U_{\theta b} / r_{b}\right)^{1 / 2}
$$

2) The characteristic functional relationships between the dimensionless radius $r^{+}$and the dimensionless intensity of swirl $\Gamma^{+}$are obtained experimentally in the forced vortex zone and in the quasi-free vortex zone respectively.

3) A calculation procedure for tangential mean velocity is proposed by making use of the abovementioned relationship between $r^{+}$and $\Gamma^{+}$and the information about the decay of $\Gamma^{*}$ in the axial direction.

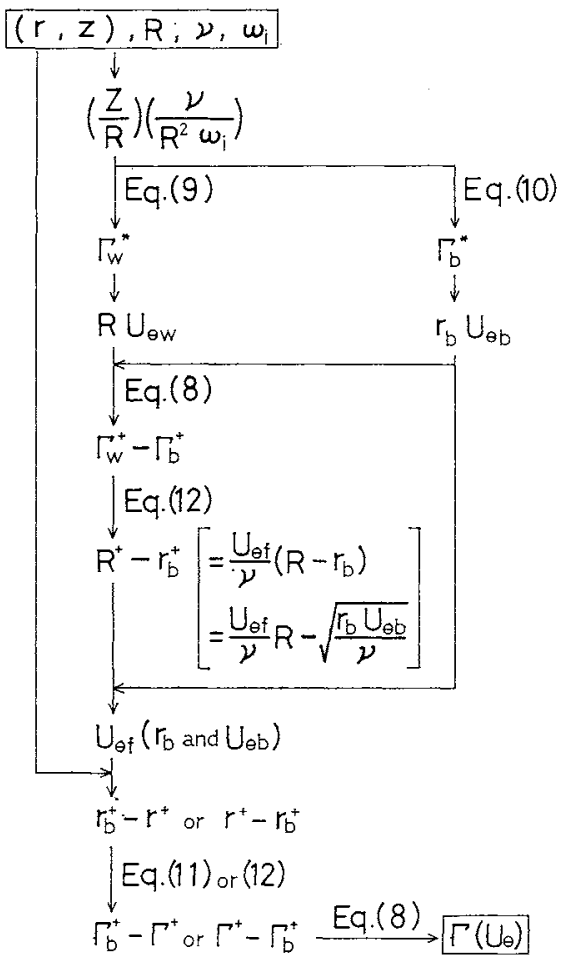

Fig. 7 Calculation procedure for tangential velocity

\section{Nomenclature}

$D$

$U_{\theta b} \quad=$ maximum value of tangential velocity $U_{\theta}$

$\begin{array}{ll}U_{\theta f} & =\text { swirl friction velocity } \\ U_{\theta w} & =\text { extrapolated value of } U_{\theta} \text { to wall }\end{array}$ [cm] $\left[\mathrm{cm}^{3} / \mathrm{s}\right]$ [cm] [-] [cm] [cm] [cm/s] $[\mathrm{cm} / \mathrm{s}]$ $[\mathrm{cm} / \mathrm{s}]$ [cm/s] $[\mathrm{cm} / \mathrm{s}]$ [cm/s] 


$\begin{array}{llr}u & =\text { velocity fluctuation } & {[\mathrm{cm} / \mathrm{s}]} \\ Z & =\text { axial directional distance between inlet } & \\ & \text { section and measured section } & {[\mathrm{cm}]} \\ z_{0} & =\text { imaginary inlet position } & {[\mathrm{cm}]} \\ & & \\ \Gamma & =\text { circulation (intensity of swirl) } & {\left[\mathrm{cm}^{2} / \mathrm{s}\right]} \\ \Gamma_{b} & =2 \pi r_{b} U_{\theta b} & {\left[\mathrm{~cm}^{2} / \mathrm{s}\right]} \\ \Gamma_{w} & =2 \pi R U_{\theta w} & {\left[\mathrm{~cm}^{2} / \mathrm{s}\right]} \\ r, \kappa & =\text { coefficient of swirl decay } & {[-]} \\ \mu & =\text { molecular viscosity } & {\left[\mathrm{g} / \mathrm{cm}^{2} \cdot \mathrm{s}\right]} \\ \nu & =\text { kinematic viscosity } & {[\mathrm{cm} / \mathrm{s}]} \\ \rho & =\text { density } & {\left[\mathrm{g} / \mathrm{cm}^{3}\right]} \\ \tau & =\text { stress } & {\left[\mathrm{g} / \mathrm{cm}^{2} \cdot \mathrm{s}^{2}\right]} \\ \left.\tau_{r \theta, b} / \mathrm{cm}^{2} \cdot \mathrm{s}^{2}\right] \\ \boldsymbol{\Omega} & =-\mu U_{\theta b} / r_{b} & {[1 / \mathrm{s}]} \\ \omega & =\text { vorticity vector } & {[1 / \mathrm{s}]} \\ \omega_{i} & =\text { component of vorticity } & {[1 / \mathrm{s}]} \\ \langle\text { Subscripts }\rangle & =\text { initial angular velocity at } z=0\left(U_{i} / R\right) & \\ b & =\text { boundary position between forced vortex } \\ & & \\ r & & \text { zone and quasi-free vortex zone } \\ z & =\text { radial direction } \\ w & =\text { axial direction } \\ \theta & =\text { wall position } \\ & =\text { tangential direction }\end{array}$

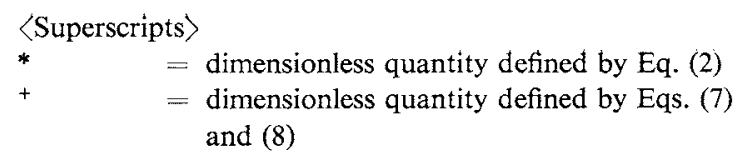

\title{
Literature Cited
}

1) Ito, S. and S. Urushiyama: Kagaku Kögaku, 32, 267 (1968).

2) Ito, S., S. Urushiyama and K. Ogawa: J. Chem. Eng. Japan, 7, 462 (1974).

3) Ito, S., K. Ogawa and C. Kuroda: Kagaku Kogaku Ronbunshu, 1, 121 (1975).

4) Ito, S., K. Ogawa and C. Kuroda: ibid., 4, 247 (1978).

5) Kreith, F. and O. K. Sonju: J. Fluid Mech., 22, 257 (1965).

6) Kubo, I. and F. C. Gouldin: Trans. ASME, J. Fluids Eng., 97, 310 (1975).

7) Murakami, M., O. Kito, Y. Katayama and Y. Iida: Trans. Soc. Mech. Eng. Jpn., 41, 1793 (1975).

8) Rochino, A. and Z. Lavan: Trans. ASME, J. Appl. Mech., 36, 151 (1969).

9) Senoo, Y. and T. Nagata: Trans. Soc. Mech. Eng. Jpn., 38, 759 (1972).

(Presented at the 11th Autumn Meeting of The Soc. of Chem. Engrs., Japan, at Tokyo October, 1977.)

\section{A METHOD OF DISPERSION COEFFICIENT MEASUREMENT}

\author{
HAJIME UNNO AND TAKASHI AKEHATA \\ Department of Environmental Chemistry and Engineering, \\ Tokyo Institute of Technology, Yokohama 227
}

\begin{abstract}
A new method of dispersion coefficient measurement is proposed, based on the measurement of mean first-passage time from an internal position to either boundary of the control region. The features of the method are as follows:

1. It can be applied to a system where the dispersion phenomenon is controlled by random motion of material.

2. Mean dispersion coefficient of a local region in a non-homogeneous system can be evaluated. There exists an optimal scale of control region for the method, and four times the scale of phenomena is recommended for the size of control region.
\end{abstract}

\section{Introduction}

The dispersion of material is fundamental for most phenomena which are dealt with in the field of both chemical and environmental engineering. The analysis or simulation of dispersion phenomena necessitates mathematical tools appropriate to its object. The dispersion model with or without plug flow $^{3,4)}$ is a

Received April 26, 1979. Correspondence concerning this article should be addressed to $H$. Unno. powerful mathematical tool for describing these phenomena, and its applications have appeared in numerous papers. Most of these papers discuss the dispersion coefficients obtained through the measurement of concentration distribution of a key component in the system.

In this paper a different method of dispersion coefficient measurement is proposed and its applicability is discussed. The method is a so-called particle-follower technique which is mathematically based on the observation of first passage time ${ }^{1}$. 